\title{
Relevance of deprivation studies in understanding rapid eye movement sleep
}

\author{
Rachna Mehta ${ }^{1,2}$ \\ Shafa Khan' \\ Birendra N Mallick' \\ 'School of Life Sciences, Jawaharlal \\ Nehru University, New Delhi, India; \\ ${ }^{2}$ Amity Institute of Neuropsychology \\ \& Neurosciences, Amity University, \\ Noida, India
}

This article was published in the following Dove Press journal: Nature and Science of Sleep

\begin{abstract}
Rapid eye movement sleep (REMS) is a unique phenomenon essential for maintaining normal physiological processes and is expressed at least in species higher in the evolution. The basic scaffold of the neuronal network responsible for REMS regulation is present in the brainstem, which may be directly or indirectly influenced by most other physiological processes. It is regulated by the neurons in the brainstem. Various manipulations including chemical, electrophysiological, lesion, stimulation, behavioral, ontogenic and deprivation studies have been designed to understand REMS genesis, maintenance, physiology and functional significance. Although each of these methods has its significance and limitations, deprivation studies have contributed significantly to the overall understanding of REMS. In this review, we discuss the advantages and limitations of various methods used for REMS deprivation (REMSD) to understand neural regulation and physiological significance of REMS. Among the deprivation strategies, the flowerpot method is by far the method of choice because it is simple and convenient, exploits physiological parameter (muscle atonia) for REMSD and allows conducting adequate controls to overcome experimental limitations as well as to rule out nonspecific effects. Notwithstanding, a major criticism that the flowerpot method faces is that of perceived stress experienced by the experimental animals. Nevertheless, we conclude that like most methods, particularly for in vivo behavioral studies, in spite of a few limitations, given the advantages described above, the flowerpot method is the best method of choice for REMSD studies.
\end{abstract}

Keywords: flowerpot method, locus coeruleus, methods of REMS deprivation, noradrenaline, stress response

\section{Introduction}

Living organisms and their constituent cells undergo basic rest and activity cycle (BRAC), which varies in phenotypic expressions. For living system(s) higher in evolution, particularly with evolved brain, BRAC has further evolved into sleep and waking. In healthy individuals, the quantity and quality of sleep and waking are expressed at optimum levels, which vary within limit under given physiological conditions. These physiological conscious states are inherently dynamic and are maintained in equilibrium with each other. Initially, sleep was considered as a passive phenomenon, which has been set aside by findings from consistent research in the mid-twentieth century. It is now accepted that sleep is an active, non-monolithic behavioral phenomenon, indispensable for optimal physiological processes including functioning of the vital organs of the body.

Based on electrophysiological parameters, sleep has been broadly classified into rapid eye movement sleep (REMS) and non-REMS (NREMS). By and large, REMS is char-
Correspondence: Birendra N Mallick School of Life Sciences, Jawaharlal Nehru University, New Delhi I I0067, India

Tel +9 I I I 26704522

Fax +9| II 26742558

Email remsbnm@yahoo.com 
acterized by desynchronized electroencephalogram (EEG), muscle atonia and rapid eye movements. In humans, REMS constitutes about $20 \%$ of total sleep time, which is considerably lesser in proportion to one $24 \mathrm{~h}$ complete day. Experimentally, it has been shown that animals, for example, $\operatorname{dogs}^{1}$ and rats, ${ }^{2,3}$ as well as humans ${ }^{4}$ not only maintain this fundamental process but also, if deprived, adequately compensate the lost REMS by its rebound increase during the post-deprivation recovery period. In the absence of such recovery, REMS deprivation (REMSD) might lead to altered states, which may cause various pathological conditions and even loss of life in extreme conditions. ${ }^{3,5}$ This means that the living beings are possessive of this state and consider REMS as non-compromisingly necessary for maintaining normal physiological processes. ${ }^{5}$ Also, the vital role of REMS may be attributed to the fact that its primary control machinery, the neural network, is located in the brainstem, where the neurons for the regulation of other vital physiological processes, for example, cardiovascular and respiratory systems, are located, and its regulation is not under voluntary control. Thus, optimum amount of sleep including REMS is important for maintaining normal and healthy living, and therefore, an understanding of this essential physiological phenomenon is necessary.

After the discovery of REMS in $1953,{ }^{6}$ consistent attempts have been made to understand its genesis, mechanism, regulation and functional significance, which have been dealt with in many reviews and scholarly articles. ${ }^{7-10}$ Several methods including transection, stimulation (electrical and chemical), lesion and microinjection into the brain, and deprivation of the subjects (humans or animals) have been carried out to understand the mechanisms of generation, regulation and functions of REMS. All these methods, particularly in vivo behavioral studies, have respective merits, and none is immune to some limitations. Like most studies, the choice of the method to be applied for investigating various aspects of REMS depends on the factor(s) whose expressions are to be explored keeping in view the limitations associated with the method to be used.

\section{Deprivation vs. induction of excess REMS}

Normally, functioning of a system along with that of its constituent components viz. cells, tissues and organs is in dynamic equilibrium leading to a healthy life. The optimum output of each of those units (individually or whole) is expressed at a lower level than the maximum that respective unit can possibly deliver. Although a cell or a system can increase its output, it cannot be higher than the maximum, and thus, the difference of maximum from that of the optimum cannot be as high as that of the difference of optimum to the minimum, which would be zero. Attempt to significantly increase the output of such a system could be nonphysiological, particularly if continued for longer duration. Also, it is likely to wear out the system very rapidly and significantly more than that of withdrawal of the output. Therefore, while designing an experiment, an increase in the expression, REMS in this case, may or may not be as significant or may be counterproductive than that of its decrease, that is, withdrawal. Further, an induction usually would need active process and may be invasive, while the withdrawal (deprivation) may not. Hence, the REMSD is the preferred method of choice for exploring, acquiring and advancing our understanding of REMS in its totality, which we review in this article. However, as there are several misapprehensions particularly related to stress associated to REMSD, the researchers face constant challenge to address this issue. We would discuss this issue for better understanding; however, before moving forward, it is important to discuss briefly the neural substrate(s) for the regulation of REMS.

\section{Brain mechanism of REMS regulation}

REMS was first identified in humans ${ }^{6}$ and was later shown in other mammals including rats, cats, dogs and so on. Its expression in species lower in evolution (particularly invertebrates) is debatable, primarily because the brain electrical activity (EEG), one of the basic characteristics for identification of REMS, is not quite evident in species possessing rudimentary or lesser evolved brain. Transection, ${ }^{11}$ lesion $^{12-14}$ and stimulation $^{15,16}$ studies identified that the dorsolateral pontine region is responsible for REMS regulation. This region possesses noradrenaline (NA)-ergic REM-OFF neurons in the locus coeruleus (LC) while cholinergic (largely) REM-ON neurons in the laterodorsal tegmentum /pedunculopontine tegmentum (LDT/PPT). ${ }^{15,17,18}$ As like the regulation by the brain of other complex behaviors and functions, a fine tuning between activation and deactivation of the REM-ON and REM-OFF neurons is necessary for the regulation of REMS. In a series of complementary studies using electrical stimulation, ${ }^{16}$ microinjection of neurotransmitter (gamma-amino butyric acid [GABA], NA, acetylcholine [ACh]) agonist or antagonist, ${ }^{19-21}$ anti-ouabain antibodies, ${ }^{22}$ activation of the wake- ${ }^{23}$ or sleep-inducing brainstem areas ${ }^{24}$ and upon REMSD, ${ }^{25}$ it was shown that if the LC REM-OFF neurons were prevented to cease activity, REMS was reduced. These findings suggest that cessation of the LC-REM-OFF neurons is prerequisite for the generation of REMS. ${ }^{26,27}$ Results from the in vivo studies suggest that GABA acting post- and presynaptically at different sites (on 
REM-OFF and REM-ON neurons) plays a significant role in inducing inhibition or withdrawal of inhibition for the regulation of REM-ON and REM-OFF neurons that regulate REMS. These studies have been reviewed in detail, and the findings have been consolidated to construct a model explaining the mechanism of regulation of REMS. ${ }^{8}$ Estimation of NA and GABA levels in different brain regions (explained later in this review) supports the model. It is difficult to experimentally show in vivo in behaving animals the pre- and postsynaptic modulation of inputs on the REM-ON and REM-OFF neurons. Therefore, as an alternative, using the in vivo data, a mathematical model was constructed, which satisfactorily explains the role of (presynaptic) withdrawal of inhibition from the REM-ON neurons, which in turn inhibits the REM-OFF neurons for the initiation of REMS. ${ }^{28}$ Also, the said model reasonably explains for the first time why REMS does not appear during waking and why it is initiated only after a period of NREMS. ${ }^{8}$ The in vivo findings from behavioral as well as REMSD studies and those from in vitro (including in silico) studies complement each other to support the proposed neural regulation of REMS.

The GABA-ergic neurons and terminals are scattered throughout the brain. ${ }^{29}$ The said model included the role of GABA-ergic neurons and terminals in the $\mathrm{LC},{ }^{30}$ in prepossitus hypoglossus, ${ }^{31}$ in substantia nigra ${ }^{32}$ and in $\mathrm{LDT} \mathrm{PPT}^{33}$ in modulating REMS. Subsequently, in recent years, isolated groups have studied the role of GABA-ergic neurons in the periaqueductal gray ${ }^{34,35}$ and in the dorsal part of the deep mesencephalic reticular nucleus, ${ }^{34,35}$ and explored their roles in REMS regulation. It is reasonable that the basic and complex physiological process like REMS would be regulated and modulated by neurons containing various neurotransmitters which are located in wider brain areas. Notwithstanding, for confirmation, it needs to be studied in behaving animals how those neurons are affected by NREMS and wake-inducing brain areas, and how the firing of those neurons is related to each other as well as with that of classically known REM-ON and REM-OFF neurons in LDT/PPT and LC, respectively, particularly in relation to modulation of REMS as has been systematically studied for classical REM-ON and REM-OFF neurons. Also, it needs to be experimentally shown if those neurons are affected by REMS loss and what role these neurons and their respective neurotransmitters play in modulating REMS loss-associated functions/symptoms, if at all.

\section{REMSD - its pros and cons}

Different approaches have been used to understand the neural regulation and functional significance of REMS, for example, a) phylogenetic or evolution studies, b) ontogenic or development studies and c) experimental studies. The first approach is based on historical observations, evidences from the literature, social and other associated changes, circumstantial evidence and their analyses. For example, among species, there are significant differences in the quality and quantity of time spent in different stages of sleep. ${ }^{36,37}$ Findings from ontogenic studies allow us to know that REMS helps proper maturation and development of the brain in particular and the nervous system at large; ${ }^{38,39}$ however, its detailed mechanism of action is not known. Maturation of the brain involves many factors including neurotransmitters. ${ }^{40}$ As REMS modulates level of NA in the brain, and NA has been reported to affect neuronal growth and development, ${ }^{41}$ it may play a significant role in REMS-associated maturation of the brain. Notwithstanding, both the phylogenetic and ontogenic studies (particularly in higher species) are slow events, time intensive and often difficult to reproduce repeatedly to prove experimentally and it is difficult to carry out planned control studies to rule out the associated nonspecific effects.

Experimental studies/approaches, on the other hand, enable us to explore the role of REMS in modulating neuronal excitability, apoptosis, cytomorphology, behavior, thermoregulation and their mechanisms. Although the physiological significance of REMS is known to a limited extent, its detailed cellular, molecular and genetic mechanisms of action need to be deciphered. Indeed, those are the subjects of active research across the globe and for which the first two methods of gathering knowledge are least helpful. Also, in vivo studies have obvious limitations to explore and understand the mechanism of effect(s) at the molecular level(s), for which often we extend studies using in vitro cells as models. Notwithstanding, almost all experimental methods are prone to some limitations, technical or otherwise. Selection of a method for the investigation of any biological process depends on the parameter to be explored and analyzed, the kind of question being raised and the suitability of the method to achieve desired results. Thus, the question remains as to what extent does the applied method is specific to induce REMSD, what are the nonspecific factors/ variables, how can they be controlled and how the effects of nonspecific factors can be eliminated so that the observed results can be correlated primarily to REMS loss alone. As such, various techniques have been used to induce REMSD; their merits and limitations are discussed below for ease of understanding. 


\section{Methods for REMSD}

\section{Flowerpot method}

The flowerpot method was first used by Jouvet et al to deprive cats, particularly of REMS. ${ }^{42}$ The novelty of this method is that it exploits a fundamental characteristic defining REMS of the experimental animal, the muscle atonia. As this method overcomes to a large extent most of the limitations encountered by other methods, it has been extensively used on experimental cats, rats and mice across the world. ${ }^{2,42}$ In this method, the animal is placed on a small island (platform) protruding over the surface of water in a tank. To rule out nonspecific effects, the control animal is placed on a slightly larger platform control (LPC) projected over surrounding water in the same room; that is, all conditions, except the platform size, remain comparable to that of the experimental animal on the smaller platform. The method derived its name from its maiden use of flowerpots by Michel Jouvet; subsequently, it has also been known as water tank, platform or pedestal method.

The success of this method depends on the ratio of platform size (both small and large) to the animal body weight. ${ }^{43}$ On the small platform, the experimental animal (though carefully) can stand, sit, move and most importantly can experience NREMS. However, at the onset of REMS due to muscle atonia, the animal tends to stretch and relax its body completely which the size of the small platform does not allow. Therefore, initially, the animal might even fall occasionally into the surrounding water and eventually (at the onset of REMS) possibly learns to wake up, often with a jerk, and saves itself from falling into the water. Although it is possible that the animal might learn to wake up before falling, it is likely that the atonia-induced jerk wakes up the animal and not the fall into the water per se. It may be compared with waking an animal with other external non-tactile stimulus, for example, sound. The primary advantage of this method is that it uses a critical REMS-associated physiological signal originating within to trigger awakening of the same animal. This may be comparable to or even better than someone waking up from sleep due to changes in blood pressure, respiratory distress, indigestion, filling of bladder and so on. Although there may not be reported quantified classical experimental verification of all these examples waking a person, from associated knowledge, we know that they affect sleep in normal and diseased individuals, for example, snoring, sleep apnea and hypertension. Also, even if the animal learns, REMS pressure due to loss of REMS overrides the learning. This is because (we have observed) if the REMSD is continued for longer period, for example, more than about 9 days (our observation), some rats might fall into the water and sometimes even fail to climb up on the platform and die of drowning. In shorter duration of REMSD, if the rats fall, they usually climb up onto the platform and save themselves.

On the larger platform, the animals enjoy both NREMS and REMS. To confirm the efficacy of the method, Mendelson et $\mathrm{al}^{2}$ continuously monitored the electrophysiological signals from the experimental and control rats for 4 days and evaluated wakefulness, NREMS and REMS. They observed that during the first $24 \mathrm{~h}$ of experiment, the experimental (on small platform) as well as LPC rats had comparable REMS, although that was significantly less than the normal home-cage control rats. Thus, although the small-platform rats are deprived of REMS even during short term ( $24 \mathrm{~h}$ deprivation), due to lack of appropriate control, the method is not suitable for short-term REMSD. However, on the fourth $24 \mathrm{~h}$ through the REMSD experiment, that is, after $72 \mathrm{~h}$ REMSD, the experimental (on small platform) rats showed significantly reduced REMS as compared to home-cage control rats as well as that of the LPC rats. Also, the rat on the smaller platform showed significantly more REMS rebound during post-REMSD recovery period than both the LPC and cage control rats. Thus, the platform method is most suitable for those studies where the animals are to be REMS deprived for $72 \mathrm{~h}$ and more.

The major drawback of the method is that the animals might experience some undesirable discomfort due to muscular overactivity, isolation, some amount of associated loss of NREMS and not complete (100\%) loss of REMS. By and large, the restricted mobility-induced effects may not differ significantly (i.e. remain comparable) on the small and large platforms. In some studies, to rule out the effects due to restricted mobility and muscular overactivity, separate control experiments were conducted where movement of animals was restricted by maintaining them in a smaller enclosure or made them to swim for varying duration, respectively. ${ }^{2,44}$ In another control group, REMS-deprived animals were allowed to recover from the lost REMS, the recovery group (REC). This experiment is carried out to confirm if the changes observed upon REMSD are reversible, particularly upon recovery of lost REMS. ${ }^{44,45}$ Nevertheless, an argument against this method is that the animals may suffer from isolation as they are kept alone in the experimental setup. However, these isolation-induced effects may be countered as the LPC animals are also maintained alone. Further, in some studies, the animals are acclimatized by keeping them isolated (alone) in their home cages prior to the start of experiment. Notwithstanding, multiple-platform method has been designed to overcome some of the drawbacks of the flowerpot method, which however, achieved limited success. 


\section{Hand arousal technique}

This method was first used by Dement, ${ }^{4}$ where a subject is awakened as and when one goes into sleep, NREMS or REMS, usually NREMS. To use this technique, one needs to record electrophysiological parameters defining sleep and waking. Based on such electrophysiological criteria if a subject is awakened at the onset of every NREMS, the subject is almost certainly deprived of total sleep (NREMS as well as REMS) as REMS does not appear unless there is some amount of NREMS. On the other hand, if the subject is awakened only at the onset of every REMS, although apparently one may be deprived mostly of REMS, practically it is difficult to continue for longer duration. This is because with increased duration of deprivation, REMS pressure builds up. This causes increased frequency of appearance of REMS leading to frequent awakening and depriving the subjects of total sleep.

To rule out nonspecific effects, another control subject/ animal (yoked control) is awakened equal number of times (as that of the experimental subject) irrespective of the former's sleep state. It was observed that the experimental subject may be selectively deprived of total sleep or REMS to a reasonable extent only if the studies are continued for a short-term deprivation (limited) period. The method is not suitable to achieve long-term (for more than several hours to a couple of days) deprivation, particularly for REMSD. This is because as the deprivation progresses, the NREMS and REMS pressure increases so much that the subject must be awakened frequently; also, the yoked control gets deprived of sleep. Besides, the method needs continuous monitoring, and therefore, if continued for longer time, the experimenter is also deprived of sleep (unless several researchers take turns, which in turn may add variability though). Use of modern computer-aided techniques has limited the human intervention; however, elaborate instrumentation and possible confounds due to instrumental use need careful planning. In this method, particularly for animal studies, electrodes need to be surgically implanted for recording the electrophysiological signals to objectively define and identify sleep and waking states.

\section{Treadmill arousal method}

In this method, the animals are maintained on a treadmill where due to continuous movement of the belt, the animals cannot sleep. ${ }^{46}$ Ideally, the animals are deprived of total sleep (NREMS as well as REMS) and not exclusively of REMS since they always remain in motion. However, in practice, after spending a while on the treadmill, the animals often learn to run in the opposite direction to that of the movement of the treadmill belt and take a nap till it reached the other end of the treadmill. By this time, to avoid falling, the animal wakes up and runs to the other end of the belt and repeats the cycle. For how long the animal may sleep depends on the speed of the treadmill belt movement, which needs to be increased with passing time to continue deprivation. It has been reported that on an average the animals could enjoy almost $40 \%$ of the time spent on treadmill in NREMS. ${ }^{47}$ In this method, the animals could neither be deprived exclusively of NREMS nor REMS. In a modification of this method, the speed of movement of the treadmill belt was regulated by the changes in the EEG so that whenever the animal under study entered REMS, the belt started rolling at varying speeds and the animal was awakened. This needed much elaborate arrangement or surgery of the animals and often faced difficulty in differentiating REMS from wakefulness only by the EEG in addition to depriving the animals of both NREMS and REMS. Also, during the experiment, free access to food and water is difficult, and appropriate control for activity needs to be designed.

\section{Pendulum method}

This technique was designed to avoid the movement restriction-associated possible stress experienced by the animals in other techniques. ${ }^{48} \mathrm{An}$ animal was kept in its home cage, which was put in a pendulum hanging from the roof and made to swing. Because of the swing, due to postural imbalance at the extremes of the swing arc, the animal was forced to remain awake to maintain balance. However, in this technique, the animal although experienced minimal REMS (0-2\%), it lost significant NREMS; the animal experienced only $19-30 \%$ of NREMS during $72 \mathrm{~h}$ of deprivation in the pendulum. The advantage of this method is that no external intervention or elaborate instrumentation is needed. However, the disadvantages of this method are that experimental animals do not get free access to food and water, simultaneous deprivation of several animals is not possible, it is difficult to design specific controls and REMS- or NREMS-specific deprivation is difficult to achieve.

\section{Multiple-platform method}

To avoid the immobilization stress experienced by the rats in flowerpot method, van Hulzen and Coenen ${ }^{49}$ developed this method in which experimental and control animals are put on platforms over water in respective tanks (as in flowerpot method) containing more than one platform (either small or large as the case may be) so that the animal could move freely 
from one platform to the other. This method was further modified, and more than one rat was kept for REMSD or control in water tanks containing many platforms ${ }^{50}$ to overcome immobilization as well as social isolation during REMSD. In this method, care is to be taken so that the platforms are neither so close to each other or to the walls of the water tank so that the animals could get support and relax easily to enjoy REMS, nor they should be too far so that the animals cannot move easily from one platform to the other.

As the animals can move across platforms and more than one animal is present in one enclosure, the immobilization and isolation stresses, if at all, are likely to be reduced. However, in practice, we have found (unpublished data, 2018) that one animal jumps onto another platform occupied by another sleeping animal and wakes the latter; that is, all the rats in the multiple small- or large-platform group are sleep deprived (including REMS) to variable duration. Although we have not recorded electrophysiological sleep-wake in these animals (which is technically challenging though), we estimated the brain Na-K ATPase activity as an estimate of REMSD (manuscript under preparation). We have reported ${ }^{44}$ that on single-platform method, the enzyme activity increases in the experimental REMS-deprived rats (on small platform) but not in the LPC rats. However, we observed that the enzyme activity increases in the multiple large platform controls (MLPCs) also and remains comparable to that of the REMSdeprived small-platform experimental rat brain as well. Thus, the finding of the MLPCs questions the effectiveness of this method. Comparable behavior of MLPCs has been mentioned in other studies, ${ }^{50}$ which may be the likely cause of differences in results in such study in addition to noncompliance with the ratio of body weight of the animals to the platform size.

\section{Rotating disc-over-water method}

In another modification, a computer-aided elaborate procedure for REMSD was designed. ${ }^{51,52}$ In this method, the experimental and control rats are simultaneously housed one on each side of a horizontally placed disk suspended over water. A vertical partition was fixed in a manner so that the disk is separated into two halves, although it could rotate unhindered. The arrangement is such that the rat on the disk would fall into the surrounding water if the disk is allowed complete rotation. The experimental and control rats are surgically implanted with electrodes for recording of electrophysiological sleep-wake. The signals are fed into a computer which gives signal to rotate the disk (platform) as and when the experimental rat goes into REMS or NREMS, as the design of experiment. As complete rotation of the disk would push the rat into the water, the animal wakes up to stop the rotation and prevents itself from going into the water below and in turn deprives itself of REMS or NREMS. Although the method overcomes the immobility stress, the control animal is awakened as and when the experimental animal goes into REMS or NREMS (as the case may be). Thus, the control animals must sleep when the experimental animal sleeps, which cannot be practically correct always. In practice, with increase in duration of deprivation period, the experimental rat frequently tends to sleep, and therefore, the control animal is also awakened frequently and is deprived of sleep; thus, the purpose is defeated. This method does not take care of isolation, it is also difficult to deprive many animals simultaneously and it needs elaborate arrangement.

We have compared all the methods of deprivation in Table 1. The flowerpot method is by far most suited under laboratory conditions to study REMSD at least in animals. The method is very effective, inexpensive, procedurally simple, does not require continuous monitoring or human or surgical intervention and allows handling several animals simultaneously. However, many experimenters and critics are uncomfortable with this method citing perceived stress experienced by the animals, which needs elaborate discussion.

\section{“Stress" vs. deprivation-induced specific effects}

"Stress" has been coined to describe exposure of an organism to a stimulus that crossed a level, which may vary in intensity and duration. The challenge is to define sub-stressful and stressful stimulus. The response of the organism depends on the environmental factor(s), type of insult (stimulus) including its quality, intensity and frequency, as well as sensitivity, predisposition, adaptation and psychosomatic state of the organism. REMSD studies are not immune to these issues. Often, REMSD studies face criticism that the observed effects may be confounded due to experimental manipulationinduced associated stress, which, if any, certainly needs to be eliminated or ruled out from the observation.

As most of the physiological processes remain in a dynamic equilibrium, it is likely that the response(s) to various exposures would vary. Therefore, it is desirable to decipher if the observed change(s) is a primary or secondary response(s). For objective analysis, it is also important to bear in mind that the observation(s) should be free from subjective bias. For example, subjectively (being emotional) by observing the behavior, particularly of a higher animal, we may be prone to raise the issue of stress as opposed to ignoring responses of cells when exposed to comparable test 


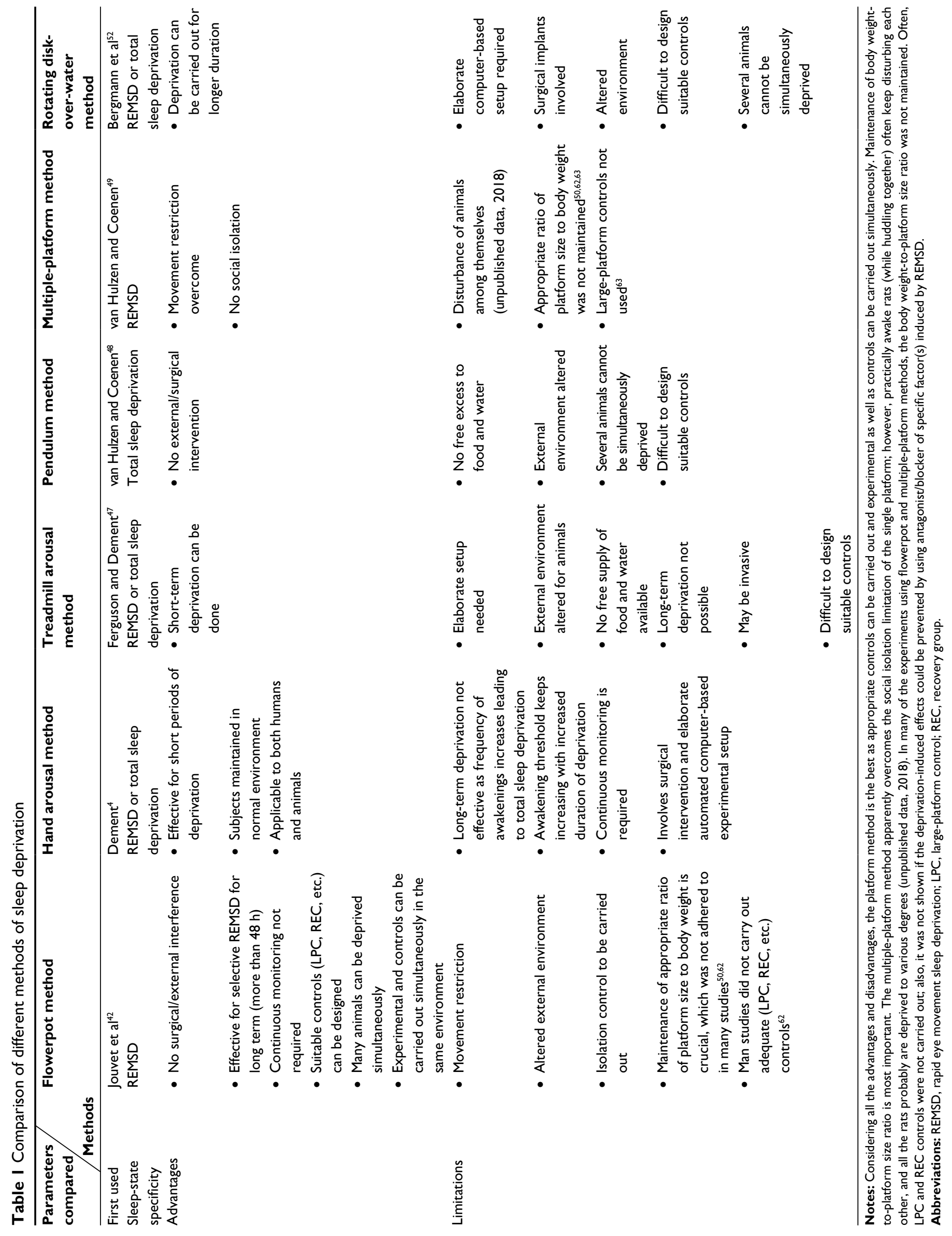


factor. Ideally, a factor (stimulus) including stressor would be experienced at the structural, molecular and cellular levels; however, their expression(s) may be ignored due to lack of our appreciation, understanding and sensitivity. On the other hand, due to the lack of appropriate definition, a response need not necessarily be categorized as stress response until its molecular mechanism of action is deciphered. Interestingly, under specific conditions, both extremes, for example, overactivity and restricted movement, isolation ${ }^{53}$ and overcrowding, ${ }^{54}$ exposure to low and high oxygenation, carbon dioxide, ${ }^{55}$ glucose ${ }^{56}$ and temperature ${ }^{57}$ and so on, may turn out to be stressful.

It needs to be distinguished if the exposure-induced effects (responses) are secondary to experimental manipulation or a direct response due to the exposure. While the former needs to be eliminated, for the rest a consistent, exclusive (marker) measurable factor is needed to define stress. Sometimes, the level of corticosterone has been taken as a marker for stress; however, it is known that corticosterone is necessary for growth and development of neurons as well as memory processing in the brain ${ }^{58-61}$ in addition to many other functions. Similarly, elevated levels of NA, adrenal and thymus weight and sympathetic overactivity have been considered as stress response. Thus, if stress response means a compensatory physiological effect, can and should that be differentiated or eliminated, and if that is attempted to differentiate, the physiological processes might react and induce some other change(s), which the observer may or may not be taking note of.

Often, we ignore variations which are statistically insignificant. However, it is possible that there could be synergism among nonsignificant changes, while antagonism among significant changes, which may appear modulating a function and/or a behavior. The nonspecific responses due to experimental intervention may be eliminated by designing appropriate control experiments. Essentially, in principle, it is comparable to eliminate noise from embedded desirable specific response by overlapping stimulus-triggered responses often by averaging repeated observations; the more are the number of observations, the better and reliable would be the averaging results (findings and associated interpretation). Thus, possible stress, if at all (not only perceived), inflicted to the animal while conducting experimental loss of either or both NREMS and REMS, needs to be countered/ eliminated by designing adequate and suitable control experiments. Alternatively, if the effects are due to (the so-called) "stressor", it needs to be shown if the responses can be prevented by blocking the "stress/stressor". In the absence of a marker, the term "stress" or "stress response" would remain in the domain of speculation and better be avoided. The best way forward will be to conduct adequate and multiple-control experiments to rule out nonspecific effects, to be as much as possible unbiased and objective in our observations.

As discussed earlier in this review, the flowerpot method allows designing appropriate control experiments to rule out various nonspecific effects; therefore, this method has been most extensively used for REMSD studies. However, citing stress, many critics favor multiple-platform method over flowerpot method (single platform). The critical point is whether appropriate controls have been carefully carried out while conducting either of the methods. In studies where multiple platforms have been used, often the critical ratio of animal weight to platform size has not been maintained. ${ }^{50,62,63}$ If such ratio is not maintained, either the experimental animals are not REMS deprived or the large-platform animals are also deprived of the desired sleep. These could account for conflicting reports of differences in the levels of corticosterone, NA, weight (body, adrenal and thymus), lipid peroxidation and so on, upon deprivation by multiple-platform method carried out by different groups. ${ }^{64-66}$

Also, in many studies, ${ }^{62,63}$ LPCs were missing, and postREMSD recovery studies were not conducted, and therefore, one cannot be sure if the observed changes were specific to REMSD. Additionally, it is desirable to show that the changes observed after REMSD can be prevented by blocking a factor induced by REMSD, that is, a causal relationship needs to be shown, which has not been conducted in most studies. ${ }^{63,65}$ Using flowerpot method, studies from this laboratory have taken care of all the issues raised above and reported consistent findings. ${ }^{67-69}$ It has also been shown that the observed changes were proportional to the duration of REMSD and the observed REMSD-induced changes initially were localized in localized (restricted) brain area and then diffused to other brain areas upon increasing the duration of REMSD. ${ }^{70}$ In support of our contention, findings from a relatively recent well-controlled study further unequivocally reinforced the efficacy of the flowerpot method for REMSD studies. ${ }^{71}$

As opposed to long-term REMSD by the flowerpot method, for short-term (4-6 h) deprivation, hand arousal may be used. However, in the latter, often there is total sleep deprivation, and it is debatable how much of the effect is due to deprivation as compared to due to other confounds (mentioned above). Also, it is debatable that if a few hours of deprivation induce some pathophysiological changes, what should be ideal control, and more importantly, whether normally body has compensatory effect for such small loss of 
REMS in particular. In such short deprivation experiments, it is not accounted for as to what proportion of total REMS is actually lost by the subjects. Also, it is not recorded normally how much REMS is present in control subjects during the clock time of conducting the study and if the subjects' REMS is dependent on the clock time. Additionally, one does not account for if the deprived subjects experience rebound increase in REMS during the rest of the $24 \mathrm{~h}$ period after the short deprivation. Therefore, it is urgently needed to innovate and design appropriate experiment for short-term REMSD. Thus, it may safely be inferred that given the complexity, the flowerpot (single-platform) method with multiple-control experiments appears to be the best possibility for conducting at least REMSD. However, any innovation particularly to reduce number of control studies and maximizing desired deprivation alone in experimental subjects will be a welcome step. Until then we, the sleep researchers, would remain indebted to Michel Jouvet for inventing the flowerpot method $^{42}$ and continue using it rather than selectively raising (biased) stress issue for some studies.

\section{Contribution of REMSD studies to understand REMS regulation and functions \\ Neural regulation of REMS}

It has been discussed earlier that transection, lesion, stimulation and pharmacological studies have contributed significantly to unravel the neural regulation of REMS. REMSD studies have complemented many of those studies to support and confirm regulation of REMS, particularly to simulate and/or extrapolate the REMSD-associated disordered states. For example, indeed upon REMSD, the REM-OFF neurons continue firing instead of cessation of activity during REMS, and REM-ON neurons tend to increase firing, possibly an attempt to compensate for REMSD ${ }^{25}$ other neurons did not show such trend. In support of the hypothesis that REMS maintains brain excitability, ${ }^{72,73}$ it has been shown by the dorsolateral pontine neurons that REMSD indeed reduced the auditory stimulation-induced evoked inhibitory responses. ${ }^{74}$

Necessity of cessation of the LC-REM-OFF neurons for induction of REMS was confirmed by increased REMS in cold-inactivated LC. ${ }^{75}$ Such studies led us to hypothesize that NA level should rise in the brain during REMSD, which has been shown later. The role of LC REM-OFF neurons and that of released NA from those neurons in inhibiting the REM-ON neurons resulting in non-appearance of REMS and inducing REMSD-induced changes was confirmed recently in in vivo experiments. In brief, it has been shown that if the NA synthesis in those neurons was prevented (downregulated by infusing si- and shRNA of TH), REMS was increased, which however was brought back to normal state if NA was microinjected into the PPT, the site of REM-ON neurons. Further, if such rats where the NA synthesis was downregulated were deprived of REMS, the REMSD-associated changes in Na-K ATPase $^{7}$ and changes in apoptosis-inducing factors were not observed. ${ }^{7}$ In a series of complementary studies if the LC REM-OFF neurons were prevented to cease activity by electrical stimulation, ${ }^{16}$ microinjection of GABA antagonist ${ }^{19-21}$ or anti-ouabain antibodies, ${ }^{22}$ REMS was reduced; that is, REMSD-like condition could be induced. Similarly, changes in the level of $\mathrm{GABA}^{68}$ and orexin ${ }^{76}$ in the brain upon REMSD support their roles in REMS and REMS loss-associated disorders (dealt with later).

\section{Behavioral changes}

After the discovery of REMS, while evaluating its physiological significance, REMSD studies were carried out in humans by Dement. ${ }^{4}$ Hand arousal was used to deprive humans of REMS; the subjects were physically awakened when during sleep they entered into state of "activated EEG", the REMS. This was perhaps the first experimental study on humans to show that lost REMS needs to be compensated and suggested an indispensable role of REMS in maintaining normal human physiological processes. ${ }^{4}$ Anxiety, irritability, increased appetite and inability to concentrate were among the other major behavioral symptoms recorded after 3-4 days of REMSD. Subjects almost uniformly showed confusion and symptoms of withdrawal.

In rats, REMSD increases body temperature in the first $24 \mathrm{~h}$ of deprivation; however, thereafter, hypothermia sets in, which if uncontrolled could lead to death. ${ }^{5}$ Other studies reported increased fighting, ${ }^{77}$ adrenocorticotropic hormoneinduced yawning, ${ }^{78}$ decreased nesting ${ }^{79}$ and postural imbalance in animals; many of the effects tended to be reversed after recovery of lost REMS. Chronic REMS restriction led to impaired physical development and anxiety-like behavior in adolescent rats, which may be attributed to alterations in the levels of NA and serotonin in amygdala and hippocampus. ${ }^{80}$ In a study to test the rate of shock avoidance, REMSD rats treated with D-amphetamine were unable to increase avoidance suggesting an impaired catecholaminergic system in REMSD rats. Catecholamine potentiating drugs like imipramine (monoamine reuptake blocker) or pargyline (suppresses monoamine degradation by monoamine oxidases [MAOs]) showed an increased retention in REMSD rats without having any effect on control rats after passive avoidance training. In 
some studies, effects of REMSD were found to be comparable to those of antidepressants. ${ }^{81}$

\section{Learning and memory}

REMS has been suggested to affect hippocampal memory processing and consolidation, ${ }^{82}$ while REMSD prevents the performance of learned tasks and impairs new learning processes; ${ }^{83}$ in other studies, sleep deprivation has been shown to affect object recognition. ${ }^{84} \mathrm{~A}$ brief exposure to $24 \mathrm{~h}$ REMSD drastically affected object recognition along with alterations in serotonin and NA levels in rat model of Parkinson's disease. ${ }^{85}$ Studies focusing on reconsolidation of memory in rats suggest that REMSD selectively disrupts consolidation, but not reconsolidation of novel object recognition. ${ }^{86}$ REMSD decreased spatial memory in Morris water maze test, while it has been shown to impair reference but not working memory in radial arm maze test. ${ }^{87}$ The effects have been shown to last for at least 2 weeks as manifested by a reduction in memory performance and by a persistently high apoptotic rate. ${ }^{88}$ Studies using a combination of behavioral, pharmacological, slice culture and optogenetic techniques showed that NA is involved in threat learning. ${ }^{89}$ In amygdala, NA acts through $\beta$-adrenergic receptors to enhance threat memory by facilitating the association between conditioned and aversive stimulus. Primary culture of rat cortical neurons showed that NA exerts neuroprotective effect against $\mathrm{A} \beta$-induced neurotoxicity $;^{90}$ these findings may help explain the beneficial effect of REMS as well as lack of adverse effects of REMSD in some studies. ${ }^{91,92}$

In humans, REMSD showed impairment of face recognition. However, it could not be correlated in these subjects whether the task performance was due to loss of REMS duration or REMS density. In another study employing slow wave sleep (SWS) deprivation and REMSD in different subjects, it was observed that SWS was essential for explicit memory consolidation for visuospatial information, while both SWS deprivation and REMSD adversely affected explicit verbal recall of the tasks. ${ }^{93}$ Notwithstanding, in some studies, REMSD could not affect emotional memory consolidation, ${ }^{91,92}$ which could be due to difference in experimental approaches and needs confirmation.

\section{Modulation of neurotransmitter levels}

Neurons exert desired effects by release of specific neurotransmitters. Also, the activities of neurons depend on the relative levels of different neurotransmitters acting on them. Isolated studies have shown that several neurotrans- mitters like NA, GABA, ACh, orexin, serotonin, histamine, dopamine and glutamate regulate REMS. Initially, based on neuronal behavior, it was predicted that NA level should be elevated in the brain upon REMSD, ${ }^{94}$ which has since been confirmed. ${ }^{68,95}$ Our recent study in rats has shown that after $96 \mathrm{~h}$ REMSD, NA increases in LC, PPT, cortex and frontal lobe, while it decreases in hippocampus. ${ }^{68}$ In the same study, we have shown that GABA decreases in LC, PPT and cortex, while it increases in hippocampus after REMSD. During recovery control, the levels of both the neurotransmitters were comparable to free moving controls. The differences in the neurotransmitter levels observed could be due to the differences in the number of projection neurons, density of synapses of the projecting neurons, number of interneurons and firing rates of all these neurons. The role of GABA in REMS regulation was proposed in rats ${ }^{21}$ and cats, ${ }^{96,97}$ which has been confirmed by actual estimation of GABA level in different brain areas in the REMSD rats. ${ }^{68}$ Similarly, REMSD-associated changes in brain acetylcholinesterase ${ }^{98}$ and orexin ${ }^{76}$ confirm their role in REMS regulation. Orexin level increased significantly in LC, PPT and hypothalamus after REMSD; the maximum increase was observed in the LC. ${ }^{76}$ This supports the dense projections of orexin-ergic neurons of posterior hypothalamus to LC. For NA-induced REMSD-associated chronic dysfunction, there should be sustained increase in NA synthesis in the brain upon REMSD. Indeed, it has been reported that the enzyme ${ }^{68,95}$ and mRNA ${ }^{95}$ of $\mathrm{TH}$, the rate-limiting enzyme involved in the biosynthesis of NA, increase in the brain after REMSD in rats.

\section{Biochemical, cellular and molecular changes}

Sleep is a behavioral phenomenon, which is affected by changes in various molecules in the brain; also, its disturbance affects almost every physiological process. Therefore, it is likely that REMS as well as REMSD must have biochemical and molecular correlates, which in turn would be responsible for REMSD-associated pathophysiological changes. REMSD has been shown to decrease both blood and brain potassium levels in rats. ${ }^{99}$ Brain glycogen (both total and free) decreases after $72 \mathrm{~h}$ of REMSD. ${ }^{100}$ Attempts to understand its effect on glucose metabolism revealed an increase in hexokinase activity (a rate-limiting enzyme in glycolysis) and a decrease in glucose 6-phosphatase activity after 4 days of REMSD in rat brain. ${ }^{101}$ During REMSD, there is an increase in all the amino acid levels in the brain with the most significant 
changes occurring in the levels of serine, glycine, alanine, phenylalanine and leucine, ${ }^{102-104}$ although no increase in protein synthesis has been observed. ${ }^{105}$ Changes in brain ammonia and nitrogen metabolism ${ }^{106}$ reflect an increased catabolism in the brain after REMSD.

We have mentioned above that REMSD elevated the level of NA, while it decreased GABA in the brain. Tyrosine hydroxylase (TH) and glutamic acid decarboxylase (GAD) are involved in synthesis of NA and GABA, respectively, while MAO-A degrades NA. Increase and decrease in the activities of the corresponding enzymes of those neurotransmitters, $\mathrm{TH}, \mathrm{MAO}-\mathrm{A}^{70}$ and $\mathrm{GAD}$, respectively, support and complement each other. ${ }^{19,31}$ Although ACh level is expected to decrease upon REMSD, its level has not yet been estimated after REMSD. A significant increase in acetylcholinesterase activity in the brain upon REMSD ${ }^{70}$ supports cholinergic downregulation during REMSD, and thus, supports ACh role in REMS regulation. Several studies in rats ${ }^{107,108}$ and in cats ${ }^{109}$ have considered increased c-fos expression in some neurons as an evidence for neuronal overactivity due to sleep/REMS loss. It has also been shown that wake-induced c-fos expression in cortical neurons is regulated by NA-ergic inputs from LC. ${ }^{110}$ However, as a critical analysis, it needs to be highlighted that c-fos is an immediate early gene, which is reported to be expressed under various conditions, ${ }^{111-113}$ and hence, the expression may not be exclusively related to sleep/REMS loss. This contention may be supported by the fact that the increased level of c-fos expression reduces with the increase in sleep/ REMS loss; that is, its level neither further increases nor remains sustained with continued REMSD. ${ }^{114}$

Excitability is a fundamental property of the neurons, and Na-K ATPase is a key factor to maintain it. REMSD has been reported to alter brain excitability ${ }^{77,115}$ and increases Na-K ATPase activity as well as expression. ${ }^{111-113}$ It has been proposed that REMSD-induced increased brain excitability is mediated by elevated NA, and thus, REMS maintains the housekeeping function of the brain. ${ }^{67}$ To understand the REMSD-associated sustained effect on brain excitability, the molecular basis of NA-induced REMSD-associated increase in Na-K ATPase activity has been investigated, and the findings support the excitability hypothesis. ${ }^{116}$ The role of $\mathrm{Ca}^{2+}$ in modulating Na-K ATPase activity and its expression has also been investigated. ${ }^{117,118}$ These changes are not nonspecific effects as there were reciprocal changes in neuronal and glial Na-K ATPase activity, and thus, help maintain homeostasis ${ }^{119}$ in the brain. In other studies, REMSD has been shown to increase phosphorylated synapsin1, which supports REMSD-associated increased vesicular mobilization and neurotransmitter release. ${ }^{120}$

\section{Cytomorphological changes}

It has been shown that REMSD-associated cytomorphologi$\mathrm{cal}^{45,121,122}$ biochemical ${ }^{67}$ and apoptotic changes ${ }^{69}$ be it in the whole brain or in localized brain regions including LC and PPT, are mediated by elevated NA. In rats, REMSD altered neuronal perimeter, area and shape in REMS-regulating areas of brain ${ }^{45}$ and induced neuronal apoptosis ${ }^{69}$ and the effects were mediated by NA. Interestingly, the changes differed depending on the functional correlates of the neurons; for example, adrenergic neurons which continue firing during REMSD increased in size, while cholinergic neurons decreased in size. These changes in neuronal cytomorphometry are likely to be the initial changes before the neurons undergo apoptosis leading to REMS loss-associated neurodegenerative disorders.

\section{Effects on epigenetic and genetic levels}

REMS is an evolutionarily conserved fundamental physiological process at least in higher animals. Therefore, it is not surprising that its loss, depending on the intensity and predisposition of the subject, affects most physiological processes in short as well as long term. The changes in the factors help explain the immediate and/or short-term effects; however, for sustained long-term effects, it is necessary that REMSD must affect at the epigenetic and genetic levels. Although this field needs serious systematic investigation, some attempts have been made in this direction. For example, continuous physical movement, which presumably caused total sleep loss in drosophila, showed changes in wide variety of gene expressions; ${ }^{123}$ some of these changes are difficult to explain though. Some studies have been conducted in rats and mice. In a recent REMSD study, changes in chromatin assembly, methylation of genes involved in learning and memory, regulation of synaptic transmission, neuronal plasticity and neuro-hypophyseal hormone synthesis in rat brain have been reported, ${ }^{124}$ which help explain REMSD-associated long-term behavioral and other changes described in this review.

\section{Neurodegeneration and associated disorders}

An increase in proapoptotic factors like Bcl2-associated death promoter protein, apoptotic protease activating factor-1, cyto- 
chrome c, caspase 9 and caspase 3 was observed in 6-day-old REMSD rats. ${ }^{69}$ The effects could be prevented by both intraperitoneal injection of $\alpha 1$-adrenoceptor antagonist, prazosin, and local microinjection of TH siRNA in LC region of rat brain, suggesting an important role of NA in the induction of REMSD-associated apoptosis. ${ }^{7}$

\section{REMS loss-associated disorders}

We have discussed above that experimental REMSD affects enzymes, ${ }^{125}$ metabolites, ${ }^{126}$ neurotransmitters, hormones, immune system including interleukins, ${ }^{127,128}$ epigenetic changes ${ }^{129}$ and neuronal structural proteins leading to apoptosis $^{69,121}$ in the brain. These would affect other somatic systems and form the basis to explain the underlying cellular and molecular mechanisms for REMSD-associated pathological conditions including in humans. Indeed, REMS and REMSD affect almost all systems in the body. An irregularity has been observed in heart rate, breathing rate, cardiac output, cardiac pressure and arterial pressure as when the subject enters REMS. ${ }^{130}$ Also, respiratory reflexes such as response to hypoxia diminish during REMS. ${ }^{131,132}$ In humans, mean blood pressure has been reported higher during REMS along with progressive decline in heart and respiratory rates. ${ }^{133,134}$ Both hemispheric and brainstem blood flow increase even higher than during wakefulness. Uncontrolled hypertensive patients have significantly reduced REMS, while REMSD contributes to hypertension and arthrosclerosis by altering blood pressure. ${ }^{135}$ Altered REMS is considered a characteristic feature of several psychosomatic and cognitive disorders. In Parkinson's disease, there is an increased latency to sleep and reduced REMS, ${ }^{136}$ while reduced latency and increased REMS have been reported in several depressed patients. ${ }^{137}$ As it is difficult to experimentally induce the disorders and study their molecular mechanism of action, we often depend on animal and cellular models to understand the mechanism of action(s).

In young rats, $96 \mathrm{~h}$ REMSD leads to decrease in homocysteine, an amino acid that is considered an independent risk factor for cardiovascular disease and stress. ${ }^{138}$ REMSD by flowerpot method induced significant elevation of blood pressure in rats with partial predisposition to developing hypertension. ${ }^{139}$ Immune responses vary in relation to sleep conditions, while immune challenge alters sleep profile. ${ }^{140}$ Sleep gets compromised ${ }^{128,140,141}$ in most infections and diseased conditions. The activity and number of natural killer cells and phagocytes decrease upon REMSD, which suggests weakened immune system. This explains why there is an increased tendency of acquiring infection, and lesions in foot, paws and gastric mucosa after total sleep deprivation and REMSD in experimental rats. ${ }^{142}$ After REMSD, the level of NA increases, and NA is also known to modulate the immune system; ${ }^{143}$ their relationship under REMS-deprived condition needs to be investigated.

In rats, Kushida ${ }^{144}$ showed that REMSD decreased plasma thyroxine (T4) and increased the ratio of plasma T3 to T4. This may explain the deficiency in growth, development and maturation of children and animals having disturbed REMS; ${ }^{145}$ however, their cause-and-effect relationship needs to be confirmed. The author also reported increased protein catabolism as evidenced by an increased level of plasma urea nitrogen. This may explain the mechanism of reduced urine $\mathrm{pH}$, metabolic acidosis, altered kidney function, urinary tract damage and finally animal death particularly upon prolonged REMSD (16-54 days). ${ }^{146}$

\section{Summary and conclusion}

REMS is an essential phenomenon which affects the overall physiology and brain functions including psychosomatic behavior. The deprivation studies have contributed immensely in understanding generation, maintenance, regulation and significance of REMS. We have collated the information gathered using the method to update our knowledge on REMS. As various methods have been used to induce REMSD, we have compared them. Because of its ease and convenience of use, minimum limitations and possibility of conducting various types of controls to rule out nonspecific effects, the flowerpot method is the method of choice for carrying out REMSD studies in animals (rats, mice and cats) across the globe. We conclude that most methods have some limitations, particularly those induced by nonspecific factors. Those nonspecific effects need to be countered and eliminated by conducting suitable and multiple-control experiments rather than ignored or overemphasized including under the category of perceived stress, if any.

\section{Acknowledgments}

RM received DST-woman scientist postdoc fellowship. SK received CSIR fellowship while conducting her PhD. BNM received funding through research grants from UGC, DBT and JC Bose fellowship and institutional umbrella support grant under the following: DBT-BUILDER; DST-PURSE and -FIST; UGC-UPEII, -DRS and Resource Networking.

\section{Disclosure}

The authors report no conflicts of interest in this work. 


\section{References}

1. O'Donnell CP, King ED, Schwartz AR, Smith PL, Robotham JL. Effect of sleep deprivation on responses to airway obstruction in the sleeping dog. J Appl Physiol. 1985;77(4):1811-1818.

2. Mendelson WB, Guthrie RD, Frederick G, Wyatt RJ. The flower pot technique of rapid eye movement (REM) sleep deprivation. Pharmacol Biochem Behav. 1974;2(4):553-556.

3. Kushida CA, Bergmann BM, Rechtschaffen A. Sleep deprivation in the rat: IV. Paradoxical sleep deprivation. Sleep. 1989;12(1): 22-30.

4. Dement W. The effect of dream deprivation. Science. 1960;131(3415): 1705-1707.

5. Jaiswal MK, Mallick BN. Prazosin modulates rapid eye movement sleep deprivation-induced changes in body temperature in rats. J Sleep Res. 2009;18(3):349-356.

6. Aserinsky E, Kleitman N. Regularly occurring periods of eye motility, and concomitant phenomena, during sleep. Science. 1953;118(3062):273-274.

7. Khanday MA, Somarajan BI, Mehta R, Mallick BN. Noradrenaline from Locus Coeruleus neurons acts on Pedunculo-Pontine neurons to prevent REM sleep and induces its loss associated effects in rats. eNeuro. 2016;3(6):1-19.

8. Mallick BN, Singh A, Khanday MA. Activation of inactivation process initiates rapid eye movement sleep. Prog Neurobiol. 2012;97(3):259-276.

9. McGinty DJ, Drucker-Colin RR. Sleep mechanisms: biology and control of REM sleep. Int Rev Neurobiol. 1982;23:391-436.

10. Siegel JM. Phylogeny and the function of REM sleep. Behav Brain Res. 1995;69(1-2):29-34.

11. Siegel JM, Tomaszewski KS, Nienhuis R. Behavioral states in the chronic medullary and midpontine cat. Electroencephalogr Clin Neurophysiol. 1986;63(3):274-288.

12. Carli G, Zanchetti A. A study of pontine lesions suppressing deep sleep in the cat. Arch Ital Biol. 1965;103(4):751-788.

13. Henley K, Morrison AR. A re-evaluation of the effects of lesions of the pontine tegmentum and locus coeruleus on phenomena of paradoxical sleep in the cat. Acta Neurobiol Exp (Wars). 1974;34(2):215-232.

14. Jones BE. Elimination of paradoxical sleep by lesions of the pontine gigantocellular tegmental field in the cat. Neurosci Lett. 1979;13(3):285-293.

15. El Mansari M, Sakai K, Jouvet M. Unitary characteristics of presumptive cholinergic tegmental neurons during the sleep-waking cycle in freely moving cats. Exp Brain Res. 1989;76(3):519-529.

16. Singh S, Mallick BN. Mild electrical stimulation of pontine tegmentum around locus coeruleus reduces rapid eye movement sleep in rats. Neurosci Res. 1996;24(3):227-235.

17. Aston-Jones G, Bloom FE. Activity of norepinephrine-containing locus coeruleus neurons in behaving rats anticipates fluctuations in the sleep-waking cycle. J Neurosci. 1981;1(8):876-886.

18. Jacobs BL. Single unit activity of locus coeruleus neurons in behaving animals. Prog Neurobiol. 1986;27(2):183-194.

19. Kaur S, Panchal M, Faisal M, Madan V, Nangia P, Mallick BN. Long term blocking of GABA-A receptor in locus coeruleus by bilateral microinfusion of picrotoxin reduced rapid eye movement sleep and increased brain Na-K ATPase activity in freely moving normally behaving rats. Behav Brain Res. 2004;151(1-2):185-190.

20. Kaur S, Saxena RN, Mallick BN. GABA in locus coeruleus regulates spontaneous rapid eye movement sleep by acting on GABAA receptors in freely moving rats. Neurosci Lett. 1997;223(2):105-108.

21. Mallick BN, Kaur S, Saxena RN. Interactions between cholinergic and GABAergic neurotransmitters in and around the locus coeruleus for the induction and maintenance of rapid eye movement sleep in rats. Neuroscience. 2001;104(2):467-485.

22. Jaiswal MK, Dvela M, Lichtstein D, Mallick BN. Endogenous ouabainlike compounds in locus coeruleus modulate rapid eye movement sleep in rats. J Sleep Res. 2010;19:183-191.
23. Thankachan S, Islam F, Mallick BN. Role of wake inducing brain stem area on rapid eye movement sleep regulation in freely moving cats. Brain Res Bull. 2001;55(1):43-49.

24. Mallick BN, Thankachan S, Islam F. Influence of hypnogenic brain areas on wakefulness- and rapid-eye-movement sleep-related neurons in the brainstem of freely moving cats. J Neurosci Res. 2004;75(1):133-142.

25. Mallick BN, Siegel JM, Fahringer H. Changes in pontine unit activity with REM sleep deprivation. Brain Res. 1990;515(1-2):94-98.

26. Pal D, Madan V, Mallick BN. Neural mechanism of rapid eye movement sleep generation: cessation of locus coeruleus neurons is a necessity. Shenq Li Xue Bao. 2005;57(4):401-413.

27. Pal D, Mallick BN. Neural mechanism of rapid eye movement sleep generation with reference to REM-OFF neurons in locus coeruleus. Indian J Med Res. 2007;125(6):721-739.

28. Kumar R, Bose A, Mallick BN. A mathematical model towards understanding the mechanism of neuronal regulation of wake-NREMSREMS states. PLoS One. 2012;7(8):e42059.

29. Berod A, Chat M, Paut L, Tappaz M. Catecholaminergic and GABAergic anatomical relationship in the rat substantia nigra, locus coeruleus, and hypothalamic median eminence: immunocytochemical visualization of biosynthetic enzymes on serial semithin plastic-embedded sections. J Histochem Cytochem. 1984;32(12):1331-1338.

30. Ali M, Jha SK, Kaur S, Mallick BN. Role of GABA-A receptor in the preoptic area in the regulation of sleep-wakefulness and rapid eye movement sleep. Neurosci Res. 1999;33(3):245-250.

31. Kaur S, Saxena RN, Mallick BN. GABAergic neurons in prepositus hypoglossi regulate REM sleep by its action on locus coeruleus in freely moving rats. Synapse. 2001;42(3):141-150.

32. Oertel WH, Tappaz ML, Berod A, Mugnaini E. Two-color immunohistochemistry for dopamine and GABA neurons in rat substantia nigra and zona incerta. Brain Res Bull. 1982;9(1-6):463-474.

33. Pal D, Mallick BN. GABA in pedunculo pontine tegmentum regulates spontaneous rapid eye movement sleep by acting on GABA-A receptors in freely moving rats. Neurosci Lett. 2004;365(3):200-204.

34. Weber F, Hoang Do JP, Chung S, et al. Regulation of REM and nonREM sleep by periaqueductal GABAergic neurons. Nat Commun. 2018;9(1):354.

35. Sapin E, Lapray D, Bérod A, et al. Localization of the brainstem GABAergic neurons controlling paradoxical (REM) sleep. PLoS One. 2009;4(1):e4272.

36. Lesku JA, Roth TC, Amlaner CJ, Lima SL. A phylogenetic analysis of sleep architecture in mammals: the integration of anatomy, physiology, and ecology. Am Nat. 2006;168(4):441-453.

37. Siegel JM. Clues to the functions of mammalian sleep. Nature. 2005;437(7063):1264-1271.

38. Marks GA, Shaffery JP, Oksenberg A, Speciale SG, Roffwarg HP. A functional role for REM sleep in brain maturation. Behav Brain Res. 1995;69(1-2):1-11.

39. Mirmiran M, Van Someren E. Symposium: normal and abnormal REM sleep regulation: the importance of REM sleep for brain maturation. J Sleep Res. 1993;2(4):188-192.

40. Manent JB, Represa A. Neurotransmitters and brain maturation: early paracrine actions of GABA and glutamate modulate neuronal migration. Neuroscientist. 2007;13(3):268-279.

41. Sanders JD, Happe HK, Bylund DB, Murrin LC. Differential effects of neonatal norepinephrine lesions on immediate early gene expression in developing and adult rat brain. Neuroscience. 2008;157(4): $821-832$

42. Jouvet D, Vimont P, Delorme F, Jouvet M. Etude de la privation selective de la phase paradoxale de sommeil chez le Chat [Study of selective deprivation of the paradoxal sleep phase in the cat]. CR Seances Soc Biol Fil. 1964;158:756-759. French [with English abstract].

43. Yanik G, Radulovacki M. REM sleep deprivation up-regulates adenosine A1 receptors. Brain Res. 1987;402(2):362-364.

44. Gulyani S, Mallick BN. Effect of rapid eye movement sleep deprivation on rat brain Na-K ATPase activity. J Sleep Res. 1993;2(1):45-50. 
45. Majumdar S, Mallick BN. Cytomorphometric changes in rat brain neurons after rapid eye movement sleep deprivation. Neuroscience. 2005;135(3):679-690.

46. Guzman-Marin R, Suntsova N, Bashir T, Nienhuis R, Szymusiak R, McGinty D. Rapid eye movement sleep deprivation contributes to reduction of neurogenesis in the hippocampal dentate gyrus of the adult rat. Sleep. 2008;31(2):167-175.

47. Ferguson J, Dement W. The effect of variations in total sleep time on the occurrence of rapid eye movement sleep in cats. Electroencephalogr Clin Neurophysiol. 1967;22(1):2-10.

48. van Hulzen ZJ, Coenen AM. The pendulum technique for paradoxical sleep deprivation in rats. Physiol Behav. 1980;25(6):807-811.

49. van Hulzen ZJ, Coenen AM. Paradoxical sleep deprivation and locomotor activity in rats. Physiol Behav. 1981;27(4):741-744.

50. Machado RB, Hipólide DC, Benedito-Silva AA, Tufik S. Sleep deprivation induced by the modified multiple platform technique: quantification of sleep loss and recovery. Brain Res. 2004;1004(1-2):45-51.

51. Rechtschaffen A, Bergmann BM. Sleep deprivation in the rat by the disk-over-water method. Behav Brain Res. 1995;69(1-2):55-63.

52. Bergmann BM, Kushida CA, Everson CA, Gilliland MA, Obermeyer W, Rechtschaffen A. Sleep deprivation in the rat: II. Methodology. Sleep. 1989;12(1):5-12.

53. Hawkley LC, Cole SW, Capitanio JP, Norman GJ, Cacioppo JT. Effects of social isolation on glucocorticoid regulation in social mammals. Horm Behav. 2012;62(3):314-323.

54. Uarquin DG, Meyer JS, Cardenas FP, Rojas MJ. Effect of overcrowding on hair corticosterone concentrations in juvenile male Wistar rats. $J$ Am Assoc Lab Anim Sci. 2016;55(6):749-755.

55. Conlee KM, Stephens ML, Rowan AN, King LA. Carbon dioxide for euthanasia: concerns regarding pain and distress, with special reference to mice and rats. Lab Anim. 2005;39(2):137-161.

56. Torres IL, Gamaro GD, Silveira-Cucco SN, et al. Effect of acute and repeated restraint stress on glucose oxidation to $\mathrm{CO}_{2}$ in hippocampal and cerebral cortex slices. Braz J Med Biol Res. 2001;34(1): 111-116.

57. Jimeno B, Hau M, Verhulst S. Strong association between corticosterone levels and temperature-dependent metabolic rate in individual zebra finches. J Exp Biol. 2017;220(23):4426-4431.

58. Barsegyan A, Mackenzie SM, Kurose BD, McGaugh JL, Roozendaal B. Glucocorticoids in the prefrontal cortex enhance memory consolidation and impair working memory by a common neural mechanism. Proc Natl Acad Sci U S A. 2010;107(38):16655-16660.

59. Chatterjee S, Sikdar SK. Corticosterone targets distinct steps of synaptic transmission via concentration specific activation of mineralocorticoid and glucocorticoid receptors. J Neurochem. 2014;128(4): 476-490.

60. Kula J, Gugula A, Blasiak A, et al. Diverse action of repeated corticosterone treatment on synaptic transmission, neuronal plasticity, and morphology in superficial and deep layers of the rat motor cortex. Pflugers Arch. 2017;469(11):1519-1532.

61. Sandi C, Rose SP. Corticosterone enhances long-term retention in oneday-old chicks trained in a weak passive avoidance learning paradigm. Brain Res. 1994;647(1):106-112.

62. Machado RB, Tufik S, Suchecki D. Role of corticosterone on sleep homeostasis induced by REM sleep deprivation in rats. PLoS One. 2013;8(5):e63520.

63. Suchecki D, Tufik S. Social stability attenuates the stress in the modified multiple platform method for paradoxical sleep deprivation in the rat. Physiol Behav. 2000;68(3):309-316.

64. Mathangi DC, Shyamala R, Subhashini AS. Effect of REM sleep deprivation on the antioxidant status in the brain of Wistar rats. Ann Neurosci. 2012;19(4):161-164.

65. Suchecki D, Lobo LL, Hipólide DC, Tufik S. Increased ACTH and corticosterone secretion induced by different methods of paradoxical sleep deprivation. J Sleep Res. 1998;7(4):276-281.
66. Süer C, Dolu N, Artis AS, Sahin L, Yilmaz A, Cetin A. The effects of long-term sleep deprivation on the long-term potentiation in the dentate gyrus and brain oxidation status in rats. Neurosci Res. 2011;70(1):71-77.

67. Mallick BN, Singh A. REM sleep loss increases brain excitability: role of noradrenaline and its mechanism of action. Sleep Med Rev. 2011;15(3):165-178.

68. Mehta R, Singh S, Khanday MA, Mallick BN. Reciprocal changes in noradrenaline and GABA levels in discrete brain regions upon rapid eye movement sleep deprivation in rats. Neurochem Int. 2017;108:190-198.

69. Somarajan BI, Khanday MA, Mallick BN. Rapid eye movement sleep deprivation induces neuronal apoptosis by noradrenaline acting on alpha1 adrenoceptor and by triggering mitochondrial intrinsic pathway. Front Neurol. 2016;7:25.

70. Thakkar M, Mallick BN. Effect of rapid eye movement sleep deprivation on rat brain monoamine oxidases. Neuroscience. 1993;55(3): 677-683.

71. McDermott CM, LaHoste GJ, Chen C, Musto A, Bazan NG, Magee JC. Sleep deprivation causes behavioral, synaptic, and membrane excitability alterations in hippocampal neurons. J Neurosci. 2003;23(29): 9687-9695.

72. Mallick BN, Thakkar M, Gulyani S. Rapid eye movement sleep deprivation induced alteration in neuronal excitability: possible role of norepinephrine. In: Mallick BN, Singh R, editors. Environment and Physiology. New Delhi: Narosa Publishing House; 1994:196-203.

73. Mallick BN, Adya HVA, Thankachan S. REM sleep deprivation alters factors affecting neuronal excitability: role of norepinephrine and its possible mechanism of action. In: Mallick BN, Inoue $\mathrm{S}$, editors. Rapid Eye Movement Sleep. Marcel Dekker Inc., New York, USA; 1999:338-354.

74. Mallick BN, Fahringer HM, Wu MF, Siegel JM. REM sleep deprivation reduces auditory evoked inhibition of dorsolateral pontine neurons. Brain Res. 1991;552(2):333-337.

75. Cespuglio R, Gomez ME, Faradji H, Jouvet M. Alterations in the sleep-waking cycle induced by cooling of the locus coeruleus area. Electroencephalogr Clin Neurophysiol. 1982;54(5):570-578.

76. Mehta R, Khanday MA, Mallick BN. REM sleep loss associated changes in orexin-A levels in discrete brain areas in rats. Neurosci Lett. 2015;590:62-67.

77. Morden B, Conner R, Mitchell G, Dement W, Levine S. Effects of rapid eye movement (REM) sleep deprivation on shock-induced fighting. Physiol Behav. 1968;3(3):425-432.

78. Lobo LL, Neumann BG, Eidman DS, Tufik S. Effects of REM sleep deprivation of ACTH-induced yawning. Pharmacology. 1990;40(3):174-178.

79. Canal-Frederick G, Quinn CP, Gillin JC, Wyatt RJ. Deprivation of rapid eye movement sleep and nesting behavior in rats. Physiol Behav. 1977;18(2):341-344.

80. da Silva Rocha-Lopes J, Machado RB, Suchecki D. Chronic REM sleep restriction in juvenile male rats induces anxiety-like behavior and alters monoamine systems in the amygdala and hippocampus. Mol Neurobiol. Epub 2017 Apr 28.

81. Maudhuit C, Jolas T, Chastanet M, Hamon M, Adrien J. Reduced inhibitory potency of serotonin reuptake blockers on central serotoninergic neurons in rats selectively deprived of rapid eye movement sleep. Biol Psychiatry. 1996;40(10):1000-1007.

82. Givens BS, Olton DS. Cholinergic and GABAergic modulation of medial septal area: effect on working memory. Behav Neurosci. 1990;104(6):849-855.

83. Pearlman C, Becker M. Brief posttrial REM sleep deprivation impairs discrimination learning in rats. Physiol Psychol. 1973;1(4):373-376.

84. Palchykova S, Winsky-Sommerer R, Meerlo P, Dürr R, Tobler I. Sleep deprivation impairs object recognition in mice. Neurobiol Learn Mem. 2006;85(3):263-271. 
85. dos Santos VV, Santos DB, Lach G, et al. Neuropeptide Y (NPY) prevents depressive-like behavior, spatial memory deficits and oxidative stress following amyloid- $\beta$ (Abeta(1-40)) administration in mice. Behav Brain Res. 2013;244:107-111.

86. Chen L, Tian S, Ke J. Rapid eye movement sleep deprivation disrupts consolidation but not reconsolidation of novel object recognition memory in rats. Neurosci Lett. 2014;563:12-16.

87. Smith CT, Conway JM, Rose GM. Brief paradoxical sleep deprivation impairs reference, but not working, memory in the radial arm maze task. Neurobiol Learn Mem. 1998;69(2):211-217.

88. Soto-Rodriguez S, Lopez-Armas G, Luquin S, et al. Rapid eye movement sleep deprivation produces long-term detrimental effects in spatial memory and modifies the cellular composition of the subgranular zone. Front Cell Neurosci. 2016;10(132):1-13.

89. Sears RM, Fink AE, Wigestrand MB, Farb CR, de Lecea L, LeDoux JE. Orexin/hypocretin system modulates amygdala-dependent threat learning through the locus coeruleus. Proc Natl Acad Sci U S A. 2013;110(50):20260-20265.

90. Madrigal JL, Kalinin S, Richardson JC, Feinstein DL. Neuroprotective actions of noradrenaline: effects on glutathione synthesis and activation of peroxisome proliferator activated receptor delta. J Neurochem. 2007;103(5):2092-2101

91. Morgenthaler J, Wiesner CD, Hinze K, Abels LC, Prehn-Kristensen A, Göder R. Selective REM-sleep deprivation does not diminish emotional memory consolidation in young healthy subjects. PLoS One. 2014;9(2):e89849.

92. Wiesner CD, Pulst J, Krause F, et al. The effect of selective REM-sleep deprivation on the consolidation and affective evaluation of emotional memories. Neurobiol Learn Mem. 2015;122:131-141.

93. Casey SJ, Solomons LC, Steier J, et al. Slow wave and REM sleep deprivation effects on explicit and implicit memory during sleep. Neuropsychology. 2016;30(8):931-945.

94. Mallick BN, Majumdar S, Faisal M, Yadav V, Madan V, Pal D. Role of norepinephrine in the regulation of rapid eye movement sleep. $J$ Biosci. 2002;27(5):539-551.

95. Porkka-Heiskanen T, Smith SE, Taira T, et al. Noradrenergic activity in rat brain during rapid eye movement sleep deprivation and rebound sleep. Am J Physiol. 1995;268(6 Pt 2):R1456-R1463.

96. Nitz D, Siegel J. GABA release in the dorsal raphe nucleus: role in the control of REM sleep. Am J Physiol. 1997;273(1 Pt 2):R451-R455.

97. Nitz D, Siegel JM. GABA release in posterior hypothalamus across sleep-wake cycle. Am J Physiol. 1996;271(6 Pt 2):R1707-R1712.

98. Thakkar M, Mallick BN. Effect of REM sleep deprivation on rat brain acetylcholinesterase. Pharmacol Biochem Behav. 1991;39(1):211-214.

99. Heiner L, Godin Y, Mark J, Mandel P. Electrolyte content of brain and blood after deprivation of paradoxical sleep. $J$ Neurochem. 1968;15(2):150-151.

100. Karadzic V, Mrsulja B. Deprivation of paradoxical sleep and brain glycogen. J Neurochem. 1969;16(1):29-34

101. Thakkar M, Mallick BN. Rapid eye movement sleep-deprivationinduced changes in glucose metabolic enzymes in rat brain. Sleep. 1993;16(8):691-694.

102. Bettendorff L, Sallanon-Moulin M, Touret M, Wins P, Margineanu I, Schoffeniels E. Paradoxical sleep deprivation increases the content of glutamate and glutamine in rat cerebral cortex. Sleep. 1996;19(1):65-71.

103. Davis JM, Himwich WA, Stout M. Cerebral amino acids during deprivation of paradoxical sleep. Biol Psychiatry. 1969;1(4):387-390.

104. Micić D, Karadzić V, Rakic LM. Changes of gamma-aminobutyric acid, glutamic acid and aspartic acid in various brain structures of cats deprived of paradoxical sleep. Nature. 1967;215(5097):169-170.

105. Bobillier P, Sakai F, Seguin S, Jouvet M. Deprivation of paradoxical sleep and in vitro cerebral protein synthesis in the rat. Life Sci. 1971;10(23):1349-1357.

106. Dostálová K, Navrátil J, Sklenovský A. Nitrogen metabolism in the CNS after paradoxical sleep deprivation in rats. Act Nerv Super. 1978;20(1):57-58.
107. Cirelli C, Pompeiano M, Tononi G. Sleep deprivation and c-fos expression in the rat brain. J Sleep Res. 1995;4(2):92-106.

108. Pompeiano M, Cirelli C, Tononi G. Effects of sleep deprivation on Fos-like immunoreactivity in the rat brain. Arch Ital Biol. 1992;130(4): 325-335.

109. Shiromani PJ, Malik M, Winston S, McCarley RW. Time course of Fos-like immunoreactivity associated with cholinergically induced REM sleep. J Neurosci. 1995;15(5 Pt 1):3500-3508.

110. Cirelli C, Pompeiano M, Tononi G. Neuronal gene expression in the waking state: a role for the locus coeruleus. Science. 1996;274(5290):1211-1215.

111. Bullitt E. Expression of C-fos-like protein as a marker for neuronal activity following noxious stimulation in the rat. JComp Neurol. 1990;296(4): $517-530$

112. Hoffman GE, Smith MS, Verbalis JG. c-Fos and related immediate early gene products as markers of activity in neuroendocrine systems. Front Neuroendocrinol. 1993;14(3):173-213.

113. Smeyne RJ, Vendrell M, Hayward M, et al. Continuous c-fos expression precedes programmed cell death in vivo. Nature. 1993;363(6425) 166-169.

114. Merchant-Nancy H, Vazquez J, Aguilar-Roblero R, Drucker-Colin R. c-fos proto-oncogene changes in relation to REM sleep duration. Brain Res. 1992;579(2):342-346.

115. Rosales-Lagarde A, Armony JL, Del Rio-Portilla Y, Trejo-Martinez D, Conde R, Corsi-Cabrera M. Enhanced emotional reactivity after selective REM sleep deprivation in humans: an fMRI study. Front Behav Neurosci. 2012;6(25):1-13.

116. Amar M, Mallick BN. Rapid eye movement sleep deprivation associated increase in Na-K ATPase activity in the rat brain is due to noradrenaline induced $\alpha 1$-adrenoceptor mediated increased $\alpha$-subunit of the enzyme. Neurochem Res. 2015;40(8):1747-1757.

117. Mallick BN, Adya HV, Faisal M. Norepinephrine-stimulated increase in Na-K ATPase activity in the rat brain is mediated through alpha1 Aadrenoceptor possibly by dephosphorylation of the enzyme. J Neurochem. 2000;74(4):1574-1578.

118. Mallick BN, Adya HV. Norepinephrine induced alpha-adrenoceptor mediated increase in rat brain Na-K ATPase activity is dependent on calcium ion. Neurochem Int. 1999;34(6):499-507.

119. Baskey G, Singh A, Sharma R, Mallick BN. REM sleep deprivationinduced noradrenaline stimulates neuronal and inhibits glial Na-KATPase in rat brain: in vivo and in vitro studies. Neurochem Int. 2009;54(1):65-71.

120. Singh S, Amar M, Mallick BN. Rapid eye movement sleep deprivation modulates synapsin I expression in rat brain. Neurosci Lett. 2012;520(1):62-66.

121. Biswas S, Mishra P, Mallick BN. Increased apoptosis in rat brain after rapid eye movement sleep loss. Neuroscience. 2006;142(2):315-331.

122. Ranjan A, Biswas S, Mallick BN. Cytomorphometric changes in the dorsal raphe neurons after rapid eye movement sleep deprivation are mediated by noradrenalin in rats. Behav Brain Funct. 2010;6(62) $1-8$.

123. Cirelli C, Bushey D. Sleep and wakefulness in Drosophila melanogaster. Ann NY Acad Sci. 2008;1129:323-329.

124. Narwade SC, Mallick BN, Deobagkar DD. Transcriptome analysis reveals altered expression of memory and neurotransmission associated genes in the REM sleep deprived rat brain. Front Mol Neurosci. 2017; 10:67.

125. Bowers MB Jr, Hartmann EL, Freedman DX. Sleep deprivation and brain acetylcholine. Science. 1966;153(3742):1416-1417.

126. Spiegel K, Leproult R, Van Cauter E. Impact of sleep debt on metabolic and endocrine function. Lancet. 1999;354(9188):1435-1439.

127. Irwin M, Thompson J, Miller C, Gillin JC, Ziegler M. Effects of sleep and sleep deprivation on catecholamine and interleukin-2 levels in humans: clinical implications. J Clin Endocrinol Metab. 1999;84(6):1979-1985.

128. Redwine L, Hauger RL, Gillin JC, Irwin M. Effects of sleep and sleep deprivation on interleukin-6, growth hormone, cortisol, and melatonin levels in humans. J Clin Endocrinol Metab. 2000;85(10):3597-3603. 
129. Mehta R, Singh A, Bókkon I, Nath Mallick B. REM sleep and its loss-associated epigenetic regulation with reference to noradrenaline in particular. Curr Neuropharmacol. 2016;14(1):28-40.

130. Fletcher EC, Gray BA, Levin DC. Nonapneic mechanisms of arterial oxygen desaturation during rapid-eye-movement sleep. J Appl Physiol Respir Environ Exerc Physiol. 1983;54(3):632-639.

131. Catterall JR, Calverley PM, MacNee W, et al. Mechanism of transient nocturnal hypoxemia in hypoxic chronic bronchitis and emphysema. J Appl Physiol (1985). 1985;59(6):1698-1703.

132. Schaeffer JI, Haddad GG. Cardiovascular response to hypoxia in puppies during natural sleep. Pediatr Res. 1984;18:344-345.

133. Snyder F, Hobson JA, Goldfrank F. Blood pressure changes during human sleep. Science. 1963;142(3597):1313-1314.

134. Snyder F, Hobson JA, Morrison DF, Goldfrank F. Changes in respiration, heart rate, and systolic blood pressure in human sleep. $J \mathrm{Appl}$ Physiol. 1964;19:417-422.

135. Andersen ML, Martins PJ, D’Almeida V, Santos RF, Bignotto M, Tufik S. Effects of paradoxica 1 sleep deprivation on blood parameters associated with cardiovascular risk in aged rats. Exp Gerontol. 2004;39(5):817-824.

136. Poryazova RG, Zachariev ZI. REM sleep behavior disorder in patients with Parkinson's disease. Folia Med (Plovdiv). 2005;47(1):5-10.

137. Palagini L, Baglioni C, Ciapparelli A, Gemignani A, Riemann D. REM sleep dysregulation in depression: state of the art. Sleep Med Rev. 2013;17(5):377-390.
138. de Oliveira AC, D’Almeida V, Hipólide DC, Nobrega JN, Tufik S. Sleep deprivation reduces total plasma homocysteine levels in rats. Can J Physiol Pharmacol. 2002;80(3):193-197.

139. Neves FA, Marson O, Baumgratz RP, et al. Rapid eye movement sleep deprivation and hypertension. Genetic influence. Hypertension. 1992;19(2 Suppl):II202-II206.

140. Opp MR. Sleeping to fuel the immune system: mammalian sleep and resistance to parasites. BMC Evol Biol. 2009;9:8-10.

141. Ruiz FS, Andersen ML, Martins RC, Zager A, Lopes JD, Tufik S. Immune alterations after selective rapid eye movement or total sleep deprivation in healthy male volunteers. Innate Immun. 2012;18(1):44-54.

142. Rechtschaffen A, Bergmann BM, Everson CA, Kushida CA, Gilliland MA. Sleep deprivation in the rat: X. Integration and discussion of the findings. Sleep. 1989;12(1):68-87.

143. Kohm AP, Sanders VM. Norepinephrine: a messenger from the brain to the immune system. Immunol Today. 2000;21(11):539-542.

144. Kushida CA. Sleep Deprivation: Clinical Issues, Pharmacology, and Sleep Loss Effects. New York: Marcel Dekker; 2005.

145. Toppila J, Asikainen M, Alanko L, Turek FW, Stenberg D, PorkkaHeiskanen T. The effect of REM sleep deprivation on somatostatin and growth hormone-releasing hormone gene expression in the rat hypothalamus. J Sleep Res. 1996;5(2):115-122.

146. Thakkar MM, McCarley RW. Physiological and neurophysiological changes. In: Kushida CA, editor. Sleep Deprivation: Basic Science, Physiology, and Behavior. New York: Marcel Dekker; 2005:265-292.
Nature and Science of Sleep

\section{Publish your work in this journal}

Nature and Science of Sleep is an international, peer-reviewed, open access journal covering all aspects of sleep science and sleep medicine, including the neurophysiology and functions of sleep, the genetics of sleep, sleep and society, biological rhythms, dreaming, sleep disorders and therapy, and strategies to optimize healthy sleep. The manuscript

\section{Dovepress}

management system is completely online and includes a very quick and fair peer-review system, which is all easy to use. Visit http://www. dovepress.com/testimonials.php to read real quotes from published authors. 